\title{
Topological Criticality on Brink of the Mott Transition in High- $T_{\mathrm{c}}$ Superconductors
}

\begin{abstract}
T.K. KOPEĆ
Institute for Low Temperature and Structure Research Polish Academy of Sciences, P.O.B. 1410, 50-950 Wrocław 2, Poland

The concept of topological excitations and the related ground state degeneracy are employed to establish an effective theory of the superconducting state evolving from the Mott insulator for high- $T_{\mathrm{c}}$ cuprates. The theory includes the effects of the relevant energy scales with the emphasis on the Coulomb interaction $U$ governed by the electromagnetic $\mathrm{U}(1)$ compact group. The results are obtained for the layered $t-t^{\prime}-t_{\perp}-U-J$ system of strongly correlated electrons relevant for cuprates. Casting the Coulomb interaction in terms of composite-fermions via the gauge flux attachment facility, we show that instanton events in the Matsubara "imaginary time", labelled by a topological winding numbers, governed by gauge flux changes by an integer multiple of $2 \pi$, are essential configurations of the phase field dual to the charge. The impact of these topological excitations is calculated for the phase diagram, which displays the "hidden" quantum critical point on verge of the Mott transition that is given by a divergence of the charge compressibility.
\end{abstract}

PACS numbers: 74.20.-z, 74.20.Fg, 71.10.Pm

\section{Introduction}

The discovery of high-temperature superconductors (HTSC) and follow-up studies of strongly correlated (SC) fermionic systems reveal that an explanation of their unusual properties appears unlikely in a way of thinking rooted in an independent electron picture. It is widely accepted that the central issue in the high temperature superconducting cuprates is physics of the doped Mott insulator [1]. There are also strong indications [2] that much of their behavior is governed by the proximity to a kind of quantum critical point (QCP). However, the resemblance to a conventional QCP is hampered by the lack of any clear signature of thermodynamic critical behavior. Usually, a QCP would be the end-point of a critical line below which an ordered phase takes place and it could be made manifested below 
the superconducting dome. Experiments appear to exclude any broken symmetry around this point although a sharp change in transport properties is observed [3]. Unfortunately, our understanding of the underlying orders in cuprates is far from being satisfactory and identifying the nature of the putative QCP is an open question. A possible way out from this difficulty would be if the degrees of freedom that rule QCP's are different from the energy degrees of freedom that govern the stable phases the critical point separates. Thus these "non-conventional" degrees of freedom could provide critical fluctuations beyond those of the order parameter fluctuations usually included in the standard Ginzburg-Landau-Wilson (GLW) description $[4,5]$. While the spontaneous symmetry breaking has become one of the main guiding principles in physics [6], there are other signatures in a physical system that are associated with the topological effects. These are instrumental for a full understanding of the physics and lead to a host of rather unexpected and exotic phenomena, which are in general of a nonperturbative nature. For example, an SC electronic system can have non-trivial topological properties which can be described by gauge fields [7]: a phase of the many-body wave function might be arbitrary but correlations among the local phases of its constituents can bring unusual gauge structures [8]. Quantum theories with topological properties have raised considerable interest in connection with a wide range of problems, among them the Aharonov-Bohm ( $\mathrm{AB}$ ) effect [9], which establishes the reality of the electromagnetic gauge potential, is a typical example.

In the present paper recognizing the significance of the $\mathrm{AB}$ non-integrable phase factor we consider the representation of strongly correlated electrons as fermions plus attached "flux tubes". Furthermore, taking into account the proper topology of the phase field dual to the charge, we recognize that the elementary excitations in strongly correlated system always carry $2 \pi$-kinks of the phase field characterized by the topological winding number. We reveal the impact of these topological excitations for the phase diagram of cuprates and show that they can induce its unusual feature: a "hidden" quantum critical point of a novel type that is not related to the symmetry breaking.

\section{Electronic model}

We consider an effective one-band electronic Hamiltonian on a tetragonal lattice that emphasizes strong anisotropy and the presence of a layered $\mathrm{CuO}_{2}$ stacking sequence in cuprates: $\mathcal{H}=\mathcal{H}_{t-J}+\mathcal{H}_{U}+\mathcal{H}_{\perp}$, where

$$
\begin{aligned}
\mathcal{H}_{t-J} & =\sum_{\alpha \ell}\left[-\sum_{\left\langle\boldsymbol{r} \boldsymbol{r}^{\prime}\right\rangle}\left(t+\mu \delta_{\boldsymbol{r}, \boldsymbol{r}^{\prime}}\right) c_{\alpha \ell}^{\dagger}(\boldsymbol{r}) c_{\alpha \ell}\left(\boldsymbol{r}^{\prime}\right)+\sum_{\left\langle\left\langle\boldsymbol{r} \boldsymbol{r}^{\prime}\right\rangle\right\rangle} t^{\prime} c_{\alpha \ell}^{\dagger}(\boldsymbol{r}) c_{\alpha \ell}\left(\boldsymbol{r}^{\prime}\right)\right] \\
& +\sum_{\ell} \sum_{\left\langle\boldsymbol{r} \boldsymbol{r}^{\prime}\right\rangle} J\left[\boldsymbol{S}_{\ell}(\boldsymbol{r}) \cdot \boldsymbol{S}_{\ell}\left(\boldsymbol{r}^{\prime}\right)-\frac{1}{4} n_{\ell}(\boldsymbol{r}) n_{\ell}\left(\boldsymbol{r}^{\prime}\right)\right] .
\end{aligned}
$$

Here $\left\langle\boldsymbol{r}, \boldsymbol{r}^{\prime}\right\rangle$ and $\left\langle\left\langle\boldsymbol{r}, \boldsymbol{r}^{\prime}\right\rangle\right\rangle$ denotes summation over the nearest-neighbor and next- 
-nearest-neighbor sites labelled by $1 \leq \boldsymbol{r} \leq N$ within the $\mathrm{CuO}$ plane, respectively, with $t, t^{\prime}$ being the bare hopping integrals $t^{\prime}>0$, while $1 \leq \ell \leq N_{\perp}$ labels copper-oxide layers. The operator $c_{\alpha \ell}^{\dagger}(\boldsymbol{r})\left(c_{\alpha \ell}(\boldsymbol{r})\right)$ creates (annihilates) an electron of $\operatorname{spin} \alpha$ at the lattice site $(\boldsymbol{r}, \ell), S_{\ell}^{a}(\boldsymbol{r})(a=x, y, z)$ stands for spin and $n_{\ell}(\boldsymbol{r})=n_{\uparrow \ell}(\boldsymbol{r})+n_{\downarrow \ell}(\boldsymbol{r})$ number operators, respectively, where $n_{\alpha \ell}(\boldsymbol{r})=$ $c_{\alpha \ell}^{\dagger}(\boldsymbol{r}) c_{\alpha \ell}(\boldsymbol{r}), \mu$ is the chemical potential and $J$ the antifferomagnetic (AF) exchange. The Hubbard term is $\mathcal{H}_{U}=\sum_{\ell \boldsymbol{r}} U n_{\uparrow \ell}(\boldsymbol{r}) n_{\downarrow \ell}(\boldsymbol{r})$ with the Coulomb energy $U$, while $\mathcal{H}_{\perp}=-\sum_{\boldsymbol{r} \boldsymbol{r}^{\prime}} t_{\perp}\left(\boldsymbol{r} \boldsymbol{r}^{\prime}\right) c_{\alpha \ell}^{\dagger}(\boldsymbol{r}) c_{\alpha \ell+1}\left(\boldsymbol{r}^{\prime}\right)$ facilitates the interlayer coupling, where $t_{\perp}$ is the interlayer hopping with $\epsilon_{\perp}\left(\boldsymbol{k}, k_{z}\right)=2 t_{\perp}(\boldsymbol{k}) \cos \left(c k_{z}\right)$, while $t_{\perp}(\boldsymbol{k})=t_{\perp}\left[\cos \left(a k_{x}\right)-\cos \left(a k_{y}\right)\right]^{2}$ as predicted on the basis of band calculations [10].

\section{Gauging out the Coulomb interaction}

We write the partition function as $Z=\int[\mathcal{D} \bar{c} \mathcal{D} \bar{c}] \mathrm{e}^{-\mathcal{S}[\bar{c}, c]}$ with the action

$$
\mathcal{S}[\bar{c}, c]=\int_{0}^{\beta} \mathrm{d} \tau\left[\sum_{\alpha \boldsymbol{r} \ell} \bar{c}_{\alpha \ell}(\boldsymbol{r} \tau) \partial_{\tau} c_{\alpha \ell}(\boldsymbol{r} \tau)+\mathcal{H}(\tau)\right]
$$

using coherent-state fermionic path integral over the Grassmann fields depending on the "imaginary time" $0 \leq \tau \leq \beta \equiv 1 / k_{\mathrm{B}} T$. Further, we write the Hubbard term in a $\mathrm{SU}(2)$ invariant way as

$$
\mathcal{H}_{U}(\tau)=U \sum_{\boldsymbol{r} \ell}\left\{\frac{1}{4} n_{\ell}^{2}(\boldsymbol{r} \tau)-\left[\boldsymbol{\Omega}_{\ell}(\boldsymbol{r} \tau) \cdot \boldsymbol{S}_{\ell}(\boldsymbol{r} \tau)\right]^{2}\right\}
$$

with charge-U(1) and spin-SU(2)/U(1) sectors, where the unit vector $\boldsymbol{\Omega}_{\ell}(\boldsymbol{r} \tau)$ sets varying in space-time spin quantization axis [11]. In the following we fix our attention on the $\mathrm{U}(1)$ invariant charge sector [12]. We consider now the following resolution of unity using the Faddeev-Popov method [13],

$$
1 \equiv \int[\mathcal{D} Q] \delta[Q-n]=\int\left[\frac{\mathcal{D} V}{2 \pi}\right] \exp \left[\mathrm{i} \sum_{\boldsymbol{r}} \int_{0}^{\beta} \mathrm{d} \tau(Q-n) V\right],
$$

where $n(\boldsymbol{r} \tau)=\sum_{\alpha} \bar{c}_{\alpha}(\boldsymbol{r} \tau) c_{\alpha}(\boldsymbol{r} \tau)$ represents the particle number, $Q(\boldsymbol{r} \tau)$ is the collective variable and $\mathrm{i} V(\boldsymbol{r} \tau)$ is fluctuating (in space and time) imaginary chemical potential conjugate to the local particle number $n_{\ell}(\boldsymbol{r} \tau)$. The field $V_{\ell}(\boldsymbol{r} \tau)$ can be written as a sum of a static $V_{0 \ell}(\boldsymbol{r})$ and periodic function $V(\boldsymbol{r} \tau)=V_{0}(\boldsymbol{r})+\tilde{V}(\boldsymbol{r} \tau)$ using the Fourier series $\tilde{V}(\boldsymbol{r} \tau)=\frac{1}{\beta} \sum_{n=1}^{\infty}\left[\tilde{V}\left(\boldsymbol{r} \omega_{n}\right) \mathrm{e}^{\mathrm{i} \omega_{n} \tau}+\right.$ c.c. $]$ with $\omega_{n}=2 \pi n / \beta$ ( $n=0, \pm 1, \pm 2$ ) being the (Bose) Matsubara frequencies. Now, we introduce the phase (or "flux") field $\phi_{\ell}(\boldsymbol{r} \tau)$ via the Faraday-type relation $\dot{\phi}_{\ell}(\boldsymbol{r} \tau) \equiv \frac{\partial \phi_{\ell}(\boldsymbol{r} \tau)}{\partial \tau}=$ $\tilde{V}_{\ell}(\boldsymbol{r} \tau)$ to remove the imaginary term i $\int_{0}^{\beta} \mathrm{d} \tau \tilde{V}_{\ell}(\boldsymbol{r} \tau) n_{\ell}(\boldsymbol{r} \tau)$ for all the Fourier modes of the $V_{\ell}(\boldsymbol{r} \tau)$ field, except for $\omega_{n}=0$ by performing the gauge transformation to the new fermionic variables $f_{\alpha \ell}(\boldsymbol{r} \tau)$, where $c_{\alpha \ell}(\boldsymbol{r} \tau)=\mathrm{e}^{\mathrm{i} \phi_{\ell}(\boldsymbol{r} \tau)} f_{\alpha \ell}(\boldsymbol{r} \tau)$, which indicates that the electron acquire a phase shift similar to that in the electric AB effect [9]. The electromagnetic U(1) group governing the phase field is compact, i.e. $\phi_{\ell}(\boldsymbol{r} \tau)$ 
has the topology of a circle $\left(S_{1}\right)$, so that topological effects can arise due to non-homotopic mappings of the configuration space onto the gauge group $S_{1} \rightarrow \mathrm{U}(1)$.

\section{Effective action with angle $U(1)$ variables}

We can now formulate the path integral for the partition function. For this we concentrate on closed paths (or world lines) in the imaginary time $\tau$ that start at position $\boldsymbol{r} \ell$ at imaginary time $\tau=0$ and end at the same position at $\tau=\beta$, which fall into distinct, disconnected (homotopy) classes labelled by the winding number [14]. Homotopically distinct paths can be summed according to various possibilities for inequivalent quantizations (superselection sectors) according to the formula

$$
\begin{aligned}
Z= & \sum_{\left\{m_{\ell}(\boldsymbol{r})\right\}} \int_{0}^{2 \pi} \prod_{\boldsymbol{r} \ell} \mathrm{d} \phi_{0 \ell}(\boldsymbol{r}) \int_{\phi_{\ell}(\boldsymbol{r} 0)=\phi_{0 \ell}(\boldsymbol{r})}^{\phi_{\ell}(\boldsymbol{r} \beta)=\phi_{\ell 0}(\boldsymbol{r})+2 \pi m_{\ell}(\boldsymbol{r})} \\
& \times \prod_{\boldsymbol{r} \ell \tau} \mathrm{d} \theta_{\ell}(\boldsymbol{r} \tau) \int[\mathcal{D} \bar{f} \mathcal{D} f] \mathrm{e}^{-\mathcal{S}[\theta, m, \bar{f}, f]}, \\
\mathcal{S}[\theta, & m, \bar{f}, f]=\sum_{\ell} \int_{0}^{\beta} \mathrm{d} \tau\left\{\frac{1}{U} \sum_{\boldsymbol{r}}\left[\frac{\partial \theta_{\ell}(\boldsymbol{r} \tau)}{\partial \tau}+\frac{2 \pi}{\beta} m_{\ell}(\boldsymbol{r})\right]^{2}\right. \\
& +\frac{2 \mu}{U} \sum_{\boldsymbol{r}} \frac{1}{\mathrm{i}}\left[\frac{\partial \theta_{\ell}(\boldsymbol{r} \tau)}{\partial \tau}+\frac{2 \pi}{\beta} m_{\ell}(\boldsymbol{r})\right] \\
& \left.+H\left[\bar{f}_{\alpha \ell}(\boldsymbol{r} \tau), f_{\alpha \ell}(\boldsymbol{r} \tau), \theta_{\ell}(\boldsymbol{r} \tau), m_{\ell}(\boldsymbol{r})\right]\right\} .
\end{aligned}
$$

The first order in time derivative term in Eq. (5) is just the topological action which does not affect the equation of motions but influences statistics [8]. The gauge transformation introduces phase factor into the hopping elements of the Hamiltonian which frustrate the motion of the fermionic subsystem. However, when charge fluctuations become phase coherent, which is signalled by $\left\langle\mathrm{e}^{\mathrm{i} \phi_{\ell}(\boldsymbol{r} \tau)}\right\rangle \neq 0$, the frustration of the kinetic energy is released. To proceed, we trace over the fermionic degrees of freedom in Eq. (5) and introduce the unimodular complex scalar $z_{\ell}(\boldsymbol{r} \tau)=\mathrm{e}^{\mathrm{i} \phi_{\ell}(\boldsymbol{r} \tau)}$, where the unimodularity constraint can be imposed with a real Lagrange multiplier $\lambda$. The partition function then becomes

$$
Z=\int\left[\mathcal{D}^{2} z\right] \prod_{\boldsymbol{r} \ell} \delta\left(\left|z_{\ell}(\boldsymbol{r} \tau)\right|^{2}-1\right) \mathrm{e}^{-\mathcal{S}\left[z, z^{\star}\right]},
$$

where

$$
\mathcal{S}\left[z, z^{\star}\right]=\frac{1}{\beta N N_{\perp}} \sum_{\boldsymbol{q} \omega_{n}} z_{\boldsymbol{q}}^{\star}\left(\omega_{n}\right) \Gamma_{\boldsymbol{q}}^{-1}\left(\omega_{n}\right) z_{\boldsymbol{q}}\left(\omega_{n}\right) .
$$

Here, $\boldsymbol{q} \equiv\left(\boldsymbol{k}, k_{z}\right)$ and, at the superconducting boundary line, the condition $\left.\Gamma_{\boldsymbol{q}=\mathbf{0}}^{-1}\left(\omega_{n}=0\right)\right|_{\lambda=\lambda_{\mathrm{c}}}=0$ fixes the Lagrange parameter while 


$$
\begin{aligned}
& \Gamma_{\boldsymbol{q}}^{-1}\left(\omega_{n}\right)=\lambda-\Sigma\left(\boldsymbol{q}, \omega_{n}\right)+\gamma^{-1}\left(\omega_{n}\right), \\
& \Sigma\left(\boldsymbol{q}, \omega_{n}\right)=\mathcal{J}_{\|}^{\prime}(\Delta) \cos \left(a k_{x}\right) \cos \left(a k_{y}\right) \\
& \quad+\sum_{\boldsymbol{q}^{\prime}} \frac{\xi_{\boldsymbol{k}^{\prime}} \Gamma_{\boldsymbol{q}+\boldsymbol{q}^{\prime}}\left(\omega_{n}\right)}{N N_{\perp}}\left[\overline{\mathcal{J}}_{\|}(\Delta)+\mathcal{J}_{\perp}(\Delta) \cos \left(c k_{z}\right)\right],
\end{aligned}
$$

where $\xi_{\boldsymbol{k}}=\cos \left(a k_{x}\right)+\cos \left(a k_{y}\right)$. Furthermore, $\gamma_{0}\left(\omega_{n}\right)$ is the Fourier transform of the bare phase correlator $\left\langle\mathrm{e}^{-\mathrm{i}\left[\phi_{\ell}(\boldsymbol{r} \tau)-\phi_{\ell^{\prime}}\left(\boldsymbol{r}^{\prime} \tau^{\prime}\right)\right]}\right\rangle_{0}$ originating from the kinetic and topological part of the action in Eq. (5). The microscopic phase stiffnesses to the lowest order in the hopping amplitudes are given by

$$
\begin{aligned}
& \mathcal{J}_{\|}(\Delta)=\frac{1}{2} \frac{t^{2}}{\beta N^{2}} \sum_{\nu_{n}}\left[\sum_{\boldsymbol{k}} \frac{|\Delta(\boldsymbol{k})|}{\nu_{n}^{2}+\bar{\mu}^{2}+|\Delta(\boldsymbol{k})|^{2}}\right]^{2}, \\
& \mathcal{J}_{\|}^{\prime}(\Delta)=-\frac{t^{\prime} \bar{\mu}}{\beta N} \sum_{\boldsymbol{k}, \nu_{n}} \frac{\cos \left(a k_{x}\right) \cos \left(a k_{y}\right)}{\nu_{n}^{2}+\bar{\mu}^{2}+|\Delta(\boldsymbol{k})|}, \\
& \mathcal{J}_{\perp}(\Delta)=\frac{1}{\beta N^{2}} \sum_{\boldsymbol{k}^{\prime} \boldsymbol{k}} \sum_{\nu_{n}} \frac{t_{\perp}^{2}\left(\boldsymbol{k}^{\prime}\right)|\Delta(\boldsymbol{k})|^{2}}{\left[\nu_{n}^{2}+\bar{\mu}^{2}+|\Delta(\boldsymbol{k})|^{2}\right]^{2}},
\end{aligned}
$$

where $\bar{\mu}=\mu-n_{f} U / 2, n_{f}=\left\langle\bar{f}_{\alpha}(\boldsymbol{r} \tau) f_{\alpha \ell}(\boldsymbol{r} \tau)\right\rangle$ is the occupation number for $f$-fermions and $\nu_{n}=\pi(2 n+1) / \beta(n=0, \pm 1, \pm 2)$ stand for the Fermi-Matsubara frequency. The stiffnesses in Eq. (9) rest on the " $d$-wave" pair amplitude $\Delta(\boldsymbol{k})=|\Delta|\left[\cos \left(a k_{x}\right)-\cos \left(a k_{y}\right)\right]$ due to the in-plane momentum space pairing of the $f$-fermions. A Gorkov-type decoupling of the AF exchange term in Eq. (1) using the valence bond operator [15] readily gives for the gap parameter

$$
1=\frac{J}{N} \sum_{\boldsymbol{k}} \frac{\left[\cos \left(a k_{x}\right)-\cos \left(a k_{y}\right)\right]^{2}}{2 \mathcal{E}_{\boldsymbol{k}}} \tanh \left(\frac{1}{2} \beta \mathcal{E}_{\boldsymbol{k}}\right)
$$

with the quasiparticle spectrum, $\mathcal{E}_{\boldsymbol{k}}^{2}=\left[\epsilon_{\|}^{\star}(\boldsymbol{k})-\bar{\mu}\right]^{2}+|\Delta(\boldsymbol{k})|^{2}$. Here, $\epsilon_{\|}^{\star}(\boldsymbol{k})$ is effective in-plane band dispersion narrowed due to the frustrated motion of the carriers in the fluctuating "bath" of U(1) gauge potentials, so that the actual tight-binding parameters are "dressed" ones $t_{X}^{\star}=t_{X}\left\langle\mathrm{e}^{-\mathrm{i}\left[\phi_{\ell}(\boldsymbol{r} \tau)-\phi_{\ell}\left(\boldsymbol{r}^{\prime} \tau\right)\right]}\right\rangle$, where $t_{X}=t, t^{\prime}, t_{\perp}$ are the bare band parameters.

\section{Results}

The resulting temperature-chemical potential phase diagram is depicted in Fig. 1. First, it shows that $T_{\mathrm{c}}$ correlates with the diagonal hopping $t^{\prime}$ in accordance with the observation that the next-nearest-neighboring hopping dominates the variation of the maximum $T_{\mathrm{c}}$ in hole doped cuprates [16]. Further, the phase diagram in Fig. 1 exhibits the special point at $\mu_{\mathrm{c}}$ defined by $2 \mu_{\mathrm{c}} / U=1 / 2$ away from the incompressible Mott state at $2 \mu_{\mathrm{c}} / U=1$ from which the superconducting lobe emanates. In cuprates there is clear evidence for the existence of a special doping point $x_{\mathrm{c}}$ in the lightly-overdoped region where superconductivity is most 


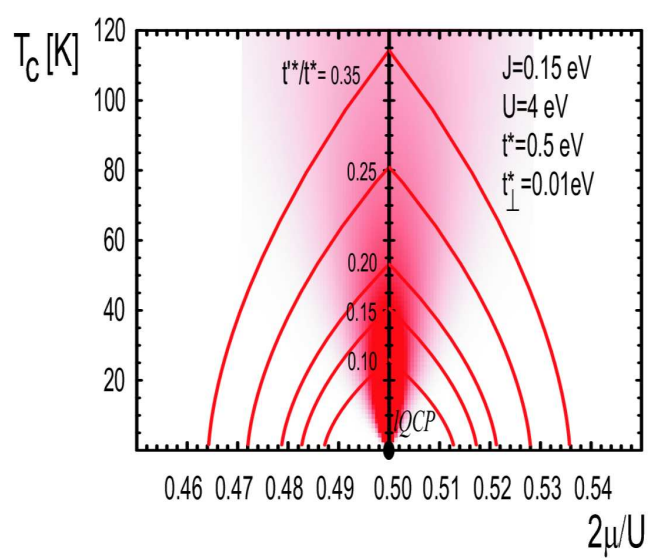

Fig. 1. The superconducting critical temperature $T_{\mathrm{c}}$ as a function of the chemical potential $\mu$ for the model's parameters as indicated in the plot. The label 1QCP marks a special point hidden in the superconducting dome, where the local charge susceptibility diverges and superconductivity is most robust. Shaded area: the density plot of the charge susceptibility $\tilde{\chi}_{\mathrm{c}}=U \chi_{\mathrm{c}} / 2$. In the "V-shaped" region fanning out to finite temperatures the electronic matter in its charge aspect is very "soft", i.e. highly compressible as opposed to the incompressible $\left(\chi_{\mathrm{c}}=0\right)$ Mott state.

robust. Such behavior indicates that this point could be a QCP while the associated critical fluctuations might be responsible for the unconventional normal state behavior [2]. Experiments appear to exclude any broken symmetry around this point although a sharp change in transport properties is observed [3] and $\partial \mu / \partial x$ becomes vanishingly small due to slow chemical potential shift implying a divergence of charge susceptibility [17]. We argue that due to topological excitations indeed such a singularity arises at $\mu_{\mathrm{c}}$ in the local charge susceptibility $\chi_{\mathrm{c}}=\partial n_{\mathrm{e}} / \partial \mu$, where $n_{\mathrm{e}} \equiv\left\langle\bar{c}_{\alpha}(\boldsymbol{r} \tau) c_{\alpha \ell}(\boldsymbol{r} \tau)\right\rangle$ is the electron filling. From Eq. (5) we readily obtain that $n_{\mathrm{e}}=n_{f}+n_{b}-2 \mu / U$, where the topological contribution is given by $n_{b}=2 \mu / U+(2 / \mathrm{i} U)\langle\dot{\phi}(\boldsymbol{r} \tau)\rangle$. In the large- $U$ limit $\mu \rightarrow n_{f} U / 2$ so that $n_{\mathrm{e}} \rightarrow n_{b}$ for strong correlations $n_{\mathrm{e}}$ is governed by the topological winding numbers rather than the number of fermionic oscillators. However, the winding number is a topologically conserved quantity and is "protected" against the small changes of $\mu$. Being an integer it cannot change at all if it has to change continuously. For substantial perturbations the ground state crosses over abruptly to other eigenstates: $n_{b}$ can change only when level degeneracies occur which happens at isolated discrete values of $2 \mu / U$. For $T=0$ we obtain for the bosonic occupation number $n_{b}=2 \mu / U-h(2 \mu / U)$, where $h(\xi+1 / 2)+1 / 2=\xi-[\xi]$, while $[\xi]$ is the greatest integer less than or equal to $\xi$. Clearly, charge susceptibility diverges at $\mu_{\mathrm{c}}$, thus marking the local QCP of a novel type, not related to the paradigm of symmetry breaking but resulting from topological effects in strongly correlated system. 


\section{Conclusions}

In the present work focusing on $t-t^{\prime}-t_{\perp}-U-J$ model it is shown that the topological excitations of charge given by the collective $\mathrm{U}(1)$ phase field in a form of "flux tubes" attached to fermions can reproduce many robust features present in the phase diagram of high- $T_{\mathrm{c}}$ cuprates, thus substantiating one of the emerging paradigms in the condensed matter physics, namely the ubiquitous competitions in strongly correlated systems. The fundamental entities that carry charge (and spin) in the copper oxides are no longer the usual Landau quasiparticles but the "flux tube" fermion composites. When charges are "liberated" then they can condense leading to superconductivity. This picture naturally leads to the pseudogap physics that is observed in the underdoped cuprates, which originates from the momentum pairing (in a $d$-wave pattern) of the fermionic parts of the electron composite controlled by the antiferromagnetic superexchange $J$. This underlines the necessity of the fundamental concept of fermion pairing in achieving the superconductivity. In particular, we found that for strong correlations the system is governed by the topological U(1) winding numbers. However, the winding number is a topologically conserved quantity and is "protected" against the small changes of system parameters. Being an integer it cannot change at all if it has to change continuously. However, changing the interaction by a large amount may cause abrupt changes in ground state properties described by different topological quantum number, which leads to a change of topological order [18]. This kind of stability might be generic for quantum systems governed by topologically non-trivial groups manifolds.

\section{References}

[1] N.F. Mott, Metal-Insulator Transitions, Taylor and Francis, London 1990.

[2] C.M. Varma, Phys. Rev. B 55, 14554 (1997); S.H. Naqib, J.R. Cooper, J.L. Tallon, C. Panagopoulos, cond-mat/0301375.

[3] T. Itoh, K. Takenaka, S. Uchida, Phys. Rev. Lett. 70, 3995 (1993).

[4] L.D. Landau, E.M. Lifshitz, Statistical Physics, Pergamon Press, Oxford 1980.

[5] K.G. Wilson, Rev. Mod. Phys. 47, 773 (1975).

[6] P.W. Anderson, Basic Notions of Condensed Matter Physics, Benjamin, New York 1984.

[7] J. Fröhlich, U. Studer, Rev. Mod. Phys. 65, 733 (1993).

[8] F. Wilczek, Fractional Statistics and Anyon Superconductivity, World Scientific, Singapore 1990.

[9] Y. Aharonov, D. Bohm, Phys. Rev. 115, 485 (1959).

[10] O.K. Andersen, A.I. Liechtenstein, O. Jepsen, F. Paulsen, J. Phys. Chem. Solids 56, 1573 (1995).

[11] Z.Y. Weng, C.S. Ting, T.K. Lee, Phys. Rev. B 43, 3790 (1991).

[12] T.K. Kopeć, Phys. Rev. B 70, 054518 (2004). 
[13] L.D. Faddeev, V.N. Popov, Phys. Lett. 25, 29 (1967).

[14] L.S. Schulman, Techniques and Applications of Path Integration, Wiley, New York 1981.

[15] G. Baskaran, Z. Zou, P.W. Anderson, Solid State Commun. 63, 973 (1987).

[16] E. Pavarini, I. Dasgupta, T. Saha-Dasgupta, O. Jepsen, O.K. Andersen, Phys. Rev. Lett. 87, 047003 (2001).

[17] N. Harima, A. Fujimori, T. Sugaya, I. Terasaki, Phys. Rev. B 67, 172501 (2003).

[18] X.-G. Wen, Int. J. Mod. Phys. B 4, 239 (1990); Adv. Phys. 44, 405 (1995). 